\title{
Modular integrated thermal control system in scientific experimental express rack on space station
}

\author{
Dongcai Guo ${ }^{1, *}$, Qiang Sheng ${ }^{1}, Y u \mathrm{Guo}^{1}$, Jing Xue ${ }^{1}$, and Ze Wang ${ }^{1}$ \\ ${ }^{1}$ Key Laboratory of Space Utilization, Technology and Engineering Center for space Utilization, \\ Chinese Academy of Sciences. Beijing 100094, China
}

Keywords: Space station, Space science experiment, Thermal system, Thermoelectric cooler, Experimental express rack.

\begin{abstract}
As an important mission of space stations, space science experiment usually requires effective temperature control measures. Scientific experimental express rack (ER) is a general design for space science experiment. In some space scientific experiments, the temperature of local target element or surrounding exceed heat sink temperature range, effective heating and cooling measures are required. Thermoelectric cooler (TEC) has high reliability and low complexity, which is applicable for temperature control in low gravity conditions. In the ER, the entire surrounding is indirectly heated or cooled by the ambient air, local target element surface is heated or cooled by liquid, TEC is thermal competent for ER thermal control attributed to the low complexity and high reliability, which can enlarge the temperature range of air and liquid. In this paper, a modular integrated thermal control system (MITCS) is designed for a specific ER, which has liquid assembly (LA), TEC assembly (TECA), heat exchanger assembly (HXA) and air cycle assembly (ACA) to provide target surface cooling and heating, entire surrounding cooling and heating. The thermal performance of MITCS using TEC are analyzed, providing guides for the design of the scientific experimental ER and other thermal systems.
\end{abstract}

\section{Introduction}

Space science is a cross-discipline of science and engineering, in the development of space science, which is researching the multilateral mechanism of various experiments under special space conditions [1]. A great deal of experimental work of basic scientific problems such as biology, physics and chemistry are required in a spaceflight [2]. At present, aerospace projects such as International Space Station has established a number of important space science research platforms, experiments in space biology, space medicine research, space fluid mechanics and other experiments are carried out and excellent results have been

\footnotetext{
* Corresponding author: guodongcai@,csu.ac.cn
} 
achieved [3]. Space science experiments is an important direction in the space activities of various countries $[4,5]$, it has an accelerated development with the entry of business space.

In the different space experiments, the temperature of experimental equipment and environment in the ER may appear different needs. Most ER is designed in a fixed operating temperature. On the other hand, some ER has a wide range of experimental application, which need a multifunctional thermal control system.

\section{Modular integrated thermal control system}

Pure water or ethylene glycol is usually used as the liquid heat sink. An internal loop system (ILS) provides liquid heat sink into the ER and transfers heat to moderate temperature loop system by heat exchanger (HX). The heat loops in space station are shown as Fig.1.

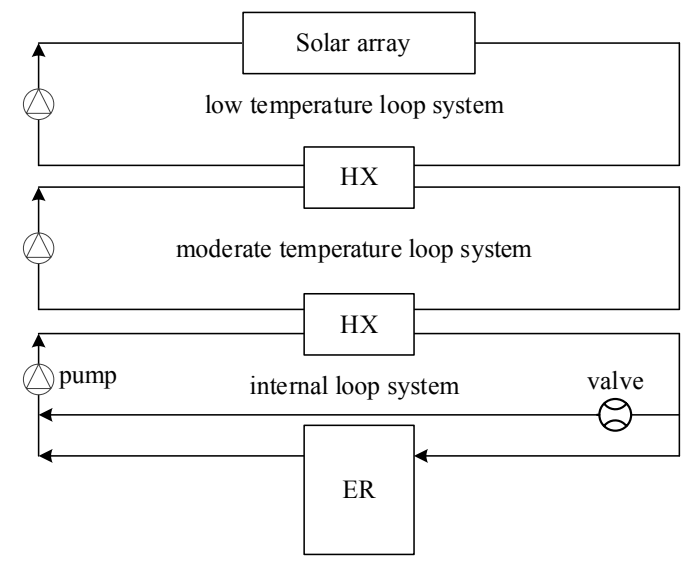

Fig. 1. Heat loops in space station.

The liquid is driven by pump in ILS and the flow rate of ER is controlled using valve on bypass.

As the thermal control requirements of ER are different, a modular integrated thermal control system (MITCS) is designed. The MITCS has different modules, including liquid assembly (LA), air cycle assembly (ACA) and heat exchanger assembly (HXA).

LA is mainly composed of flexible pipes, quick connects (QC), cold plates. QC could be disconnected to QC body and QC stem [6]. When body and stem is disconnected, both of them are sealed. QC is widely used in liquid loop for the replacement of modules, in this paper it is used for the disassembly of liquid loop without leakage. When an experiment is over, modules of LA could be quick disconnected with none leakage, then stored in a certain rack using less volume. 


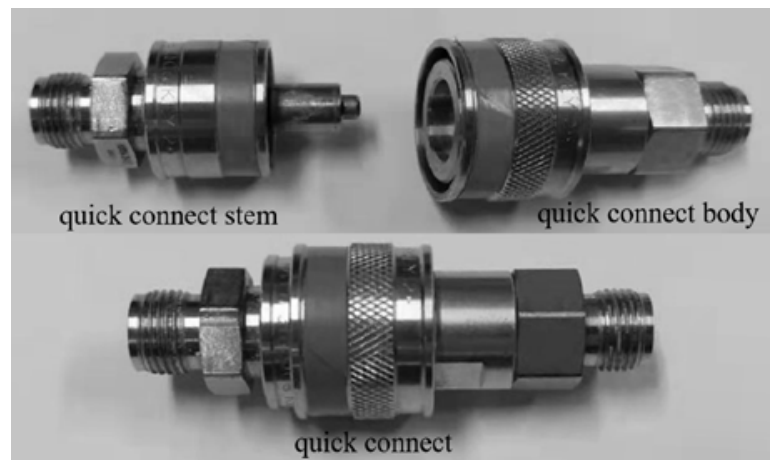

Fig. 2. Quick connect.

ACA is mainly composed of fan, air duct. HXA has connect fittings for LA and ACA, heat is transferring from air to liquid. LA, ACA and HXA could be used for thermal control in some experimental applications, which is shown in Fig.3. All of the assembly is fixed at base plate of ER. When an assembly is combined with another, there are fixtures in the interface.

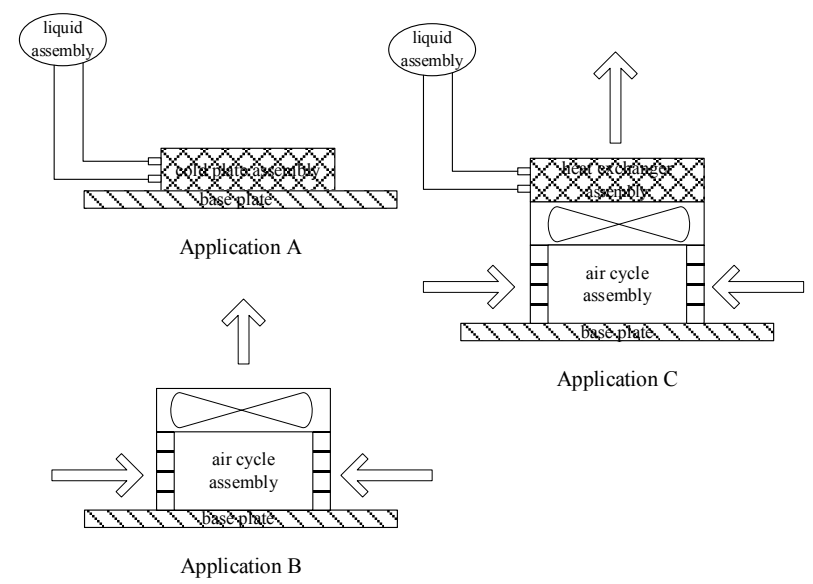

Fig. 3. Application of LA, ACA and HXA

Application A is for the need of specifically local targets, such as electronic board and electronic control box with high heat generation. Heat would be conducted from targets to cold plate, then transferred to liquid in LA.

Application B is for air cycle in ER, air is driven by fan. When air also needs cooling, LA and ACA could be combined as Application C shown in Fig.3. Air is cooling by liquid in heat exchanger.

\section{Enlarging applicable temperature range by thermoelectric cooler}

It is seen that basic heat sink of Application A and C is both liquid in ILS. Accordingly, the temperature of target element and surrounding is limited by the temperature of ILS. However, the temperature requirements vary greatly among different space experiments, which may exceed the temperature range of the basic heat sink. Therefore, it is necessary to design a general temperature control method to satisfy different temperature requirements with a heat sink working in a relatively narrow temperature range. 
Many refrigeration technologies commonly applied on ground may not be suitable for the special conditions of space. Taking the most common vapor compressive refrigerator using Freon for example, the existence of high-speed rotary compressor and the potential leakage of high-pressure refrigerant are harmful to the reliability of the whole system, although its coefficient of performance $(C O P)$ is quite outstanding. R134a is considered as the most promising refrigerant used in spacecraft attributed to its relatively low working pressure [7], but the discharge pressure of compressor still exceeds 10 bar. Moreover, compared to terrestrial conditions, the two-phase heat transfer intensity is deteriorated under microgravity as the development of thermal boundary layer is restrained $[8,9]$.

Comparatively, thermoelectric cooler (TEC) is powered by electricity without any moving parts and refrigerant. Correspondingly, there is no risk of leakage which may cause pollution to the space station environment $[10,11]$. Besides, TEC has the merits of non-noise and high integration $[12,13]$. There are already extensive applications of TEC in space experiment like biologic sample separation [14]. TEC has also been successfully applied to the modular equipment, such as space telescope infrared camera [15], quartz crystal microbalance [16], membrane evaporator [17], etc. For these reasons, TEC is chosen as the refrigerator in MITCS.

TEC assembly (TECA) is composed of thermoelectric cooler and cold plates. Two cold plates were appressed on the TEC cold and hot side. Liquid flows and TECA and splits in two parts, which is cooled in cold side or heated in hot side by TEC.

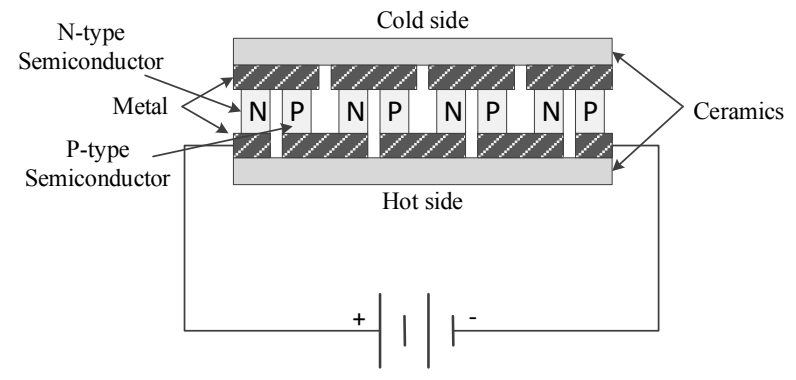

Fig. 4. Structure of TECA.

TECA could be added in application A and application $\mathrm{C}$ to enlarge the applicable temperature range, which is shown in Fig.5. In application D, the TEC attached on the surface of the equipment releases the heat to the liquid. In application E, the basic heat sink is precooled in TECA, the circulated air in the ER is cooled in HXA, creating the required thermal ambience for the space experiment. When to temperature requirement is higher than heat sink, the cold and heat side of TECA could be transposed to achieve heating capacity. 


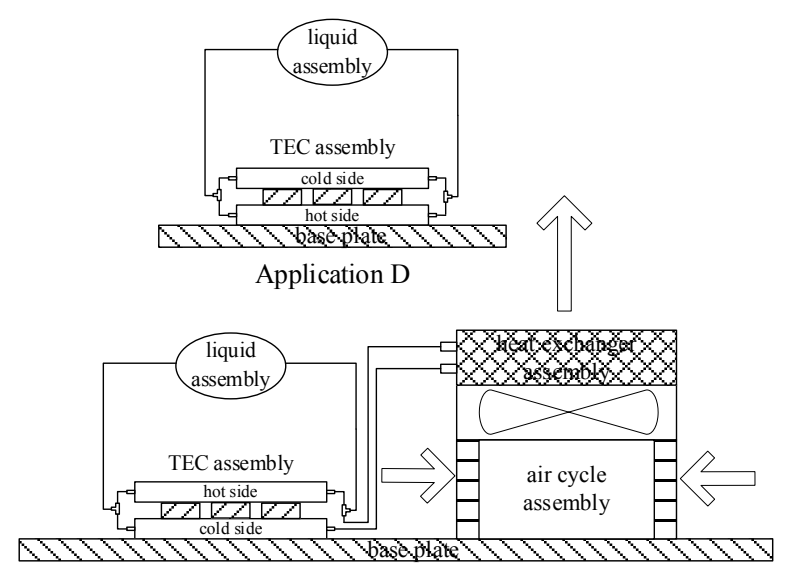

Application F

Fig. 5. Application of TECA.

\section{Effects of TECA}

Thermoelectric cooler is a refrigeration technology based on Seebeck effect, Peltier effect, Joule effect, Thomson effect and Fourier effect. The dominant effect is Peltier effect, followed by the Joule effect and Fourier effect [18]. As shown in Fig.4, a thermoelectric unit connects several p-type and n-type semiconductors by using metal plates as conductors. When this unit is electrified, heat will be transferred from the cold side to the hot side. Ceramics are arranged on both sides on metal plates as the electricity insulation layers. The principle of heat transfer process is shown in Fig.6.

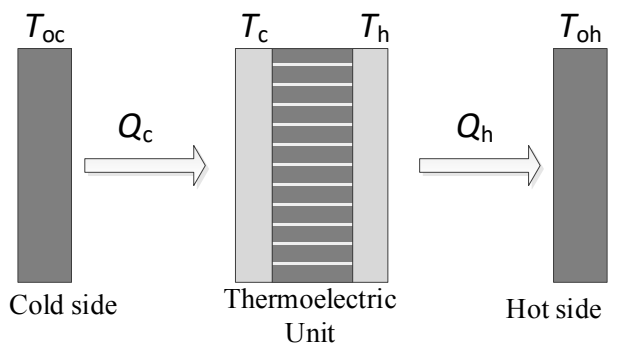

Fig. 6. Heat transfer in TEC.

The heat transfer of thermoelectric module can be then described as the follows.

$$
\begin{aligned}
& \mathrm{Q}_{\mathrm{c}}=\left(\mathrm{T}_{\mathrm{oc}}-\mathrm{T}_{\mathrm{c}}\right) / \mathrm{L}_{\mathrm{c}} \\
& \mathrm{Q}_{\mathrm{h}}=\left(\mathrm{T}_{\mathrm{h}}-\mathrm{T}_{\mathrm{oh}}\right) / \mathrm{L}_{\mathrm{h}} \\
& \mathrm{Q}_{\mathrm{c}}=\alpha \mathrm{IT}_{\mathrm{c}}-0.5 \mathrm{I}^{2} \mathrm{R}-\mathrm{K}\left(\mathrm{T}_{\mathrm{h}}-\mathrm{T}_{\mathrm{c}}\right) \\
& \mathrm{Q}_{\mathrm{h}}=\alpha \mathrm{IT}_{\mathrm{h}}+0.5 \mathrm{I}^{2} \mathrm{R}-\mathrm{K}\left(\mathrm{T}_{\mathrm{h}}-\mathrm{T}_{\mathrm{c}}\right) \\
& \mathrm{P}=\mathrm{UI}=\alpha \mathrm{I}\left(\mathrm{T}_{\mathrm{h}}-\mathrm{T}_{\mathrm{c}}\right)+\mathrm{I}^{2} \mathrm{R}
\end{aligned}
$$

where, $\mathrm{Q}_{\mathrm{c}}$ is the cooling capacity, $\mathrm{Q}_{\mathrm{h}}$ is the heating capacity, $\mathrm{T}_{\mathrm{c}}$ is the temperature of cold side, $\mathrm{T}_{\mathrm{h}}$ is the temperature of hot side, $\alpha$ is Seebeck coefficient, $\mathrm{R}$ is resistance of thermoelectric 
cooler module, $\mathrm{K}$ is heat conductivity coefficient of thermoelectric cooler module, $\mathrm{L}_{\mathrm{c}}$ is thermal resistance of cold side, $\mathrm{L}_{\mathrm{h}}$ is thermal resistance of hot side, $\mathrm{T}_{\mathrm{oc}}$ is the temperature of cold media, $\mathrm{T}_{\mathrm{oh}}$ is the temperature of hot media. In Eq.3, the first part represents Peltier effect, the second part represents Joule effect, and the third part represents Fourier effect [19].

The cooling capacity of TECA in Application D or E is shown in Fig.7. In this paper, ethylene glycol solution is used as heat sink in ILS, whose temperature is generally in the range of 17 to $26{ }^{\circ} \mathrm{C}$. The cooling capacity increases first and then decrease, which is meaning that the TECA is possible to run with best heat capacity. Joule effect increases faster than Peltier effect with current, leading to the decrease of derivative of cooling capacity curve, which is also seen from Eq. (3).

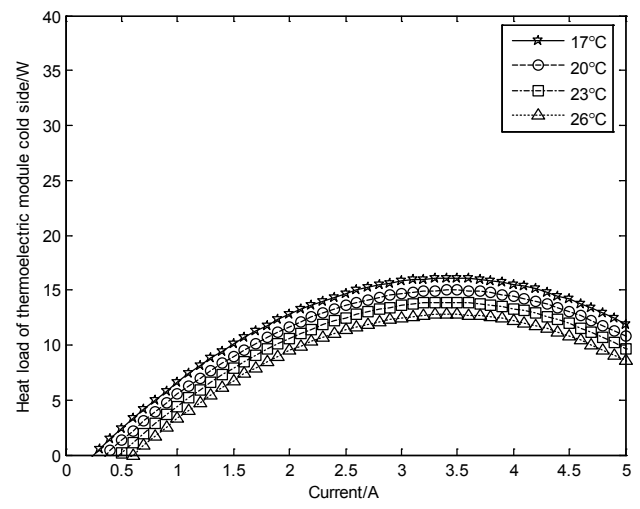

Fig. 7. Effects of current on cooling capacity at various heat sink temperature.

The TECA is designed for an experimental requirement with largest cooling capacity, the current is fixed at the optimal working condition. When another experiment needs a lower cooling capacity, pulse width modulation (PWM) control mode could be used for a certain current with best cooling capacity.

\section{Summary}

The modules of MITCS have characteristics of simple structure and flexibility, which can achieve functional scalability by combining each other. MITCS does not need a fixed position in the spacecraft, so it can be decentralized storage avoiding the structural strength requirements during the launch spacecraft. Key findings from the study are summarized below.

(1) MITCS could provide target surface cooling and heating, entire surrounding cooling and heating, respectively.

(2) Sealing of liquid interfaces between different modules is an important consideration for MITCS. Quick connect can ensure rapid disassembly of the MITCS while ensuring none leakage.

(3) Thermoelectric cooler has advantages of high reliability free of moving parts, which is suitable for temperature control in MITCS. In this paper, TECA is used to extend the range of temperature control. The greatest cooling capacity of the TECA exists under cooling mode, so the TECA should be designed in the optimal current and controlled by PWM mode. 


\section{References}

1. Brinckmann E. ESA hardware for plant research on the International Space Station. Advances in Space Research. 36 (7) (2005) 1162-1166.

2. Barmin I, Egorov A, Senchenkov A, Filatov I, Bryukhanov N, Tsvetkov V. Utilization of the "Progress" transport spacecraft as an element of the International Space Station for experiments under mu g-conditions. First International Symposium on Microgravity Research \& Applications in Physical Sciences and Biotechnology. 454 (2000) 1039-1044.

3. NASA. Interfacial behaviors and Heat transfer characteristics in Boiling Two-Phase Flow.

https://www.nasa.gov/mission_pages/station/research/experiments/explorer/Investigati on.html?\#id=1034.

4. Ishioka N, Suzuki H, Asashima M, Kamisaka S, Mogami Y, et al. Development and verification of hardware for life science experiments in the Japanese experiment module "Kibo" on the International Space Station. Journal of Gravitational Physiology: A Journal of the International Society for Gravitational Physiology 11 (1) (2004) 81-91.

5. Larson M, Croonquist A, Dick GJ, Liu YM. The science capability of the Low Temperature Microgravity Physics Facility. Physica B-condensed Matter 329 (2003) 1588-1589.

6. Quick connects. https://www.swagelok.com.cn/en/catalog/Quick-Connects

7. S.H. Lee, I. Mudawar, M.M. Hasan, Thermal Analysis of Hybrid Single-Phase, Two-Phase and Heat Pump Thermal Control System (Tcs) for Future Spacecraft, Applied Thermal Engineering, 100 (2016) 190-214.

8. M.T. Lebon, C.F. Hammer, J. Kim, Gravity Effects on Subcooled Flow Boiling Heat Transfer, International Journal of Heat and Mass Transfer, 128 (2019) 700-714.

9. H. Ohta, Microgravity Heat Transfer in Flow Boiling, Advances in heat transfer, 37 (2003) 1-76

10. M. Zebarjadi, K. Esfarjani, M.S. Dresselhaus, Z. Ren, G. Chen, Perspectives on Thermoelectrics: From Fundamentals to Device Applications, Energy and Environmental Science, 5 (2012) 5147-5162.

11. E. Alleno, N. Lamquembe, R. Cardosogil, M. Ikeda, F. Widder, O. Rouleau, C. Godart, Y. Grin, S. Paschen, A Thermoelectric Generator Based on an N-Type Clathrate and a P-Type Skutterudite Unicouple, Physica Status Solidi A-applications and Materials Science, 211 (2014) 1293-1300.

12. N.P. Semena, The Features of Application of Thermoelectric Converters in Spacecraft Systems of Temperature Control, Thermophysics and Aeromechanics, 20 (2013) 211-222.

13. K. Gaurav, S.K. Pandey, Efficiency Calculation of a Thermoelectric Generator for Investigating the Applicability of Various Thermoelectric Materials, Journal of Renewable and Sustainable Energy, 9 (2017) 014701.

14. T.S. Topham, G.E. Bingham, H. Latvakoski, I. Podolski, V.S. Sychev, A. Burdakin, Observational Study: Microgravity Testing of a Phase-Change Reference on the International Space Station, npj Microgravity, 1 (2015) 15009.

15. J.A. Morales de los Ríos, E. Joven, L. del Peral, M. Reyes, J. Licandro, M.D. Rodríguez Frías, The Infrared Camera Prototype Characterization for the Jem-Euso Space Mission, 
Nuclear Instruments and Methods in Physics Research Section A: Accelerators, Spectrometers, Detectors and Associated Equipment, 749 (2014) 74-83.

16. G. Bale, A. Holland, P. Seller, B. Lowe, Cooled Cdznte Detectors for X-Ray Astronomy, Nuclear Instruments and Methods in Physics Research Section A: Accelerators, Spectrometers, Detectors and Associated Equipment, 436 (1999) 150-154.

17. J.H. Miernik, B.H. Shah, C.F. Mcgriff, Waste Water Processing Technology for Space Station Freedom - Comparative Test Data Analysis, in: international conference on evolvable systems, 1991, pp. 229-240.

18. Bugby D, Zimbeck W, Kroliczek E. Thermal Management Architecture for Future Responsive Spacecraft. Space, Propulsion \& Energy Sciences International Forum Spesif 1103 (2009) 30-38.

19. Zhao J, Zhu HL, Qiu HB, Zhuang J, Yu HF. Numerical Simulation and Experimental Research on Multistage Thermoelectric Refrigeration. Chinese Journal of Refrigeration Technology 35 (4) (2015) 17-21. 